\title{
Monitoring and modelling of cryptocurrency trend resistance by recurrent and R/S-analysis
}

\author{
Hanna Danylchuk ${ }^{1, *}$, Oksana Kovtun $^{2}$, Liubov Kibalnyk ${ }^{1}$, and Oleksii Sysoiev ${ }^{3}$ \\ ${ }^{1}$ Bohdan Khmelnytsky National University of Cherkasy, Department of Economics and Business Modelling, Cherkasy, Ukraine \\ ${ }^{2}$ University of Educational Management, Department of Public Administration and Project Management, Kyiv, Ukraine \\ ${ }^{3}$ Kyiv International University, Department of Economics, Management, Business, Kyiv, Ukraine
}

\begin{abstract}
The paper focuses on monitoring and modelling of the cryptocurrency market. The application of the chosen research methods is based on the analysis of existing methods and tools of economic and mathematical modelling of time series research on the example of the cryptocurrency market. It is proved that the use of individual methods is not relevant, as they do not give an adequate assessment of the specified market, so a comprehensive approach is the most acceptable. Therefore, monitoring and modelling of some cryptocurrency pairs with different capitalization degree were implemented by fractal and recurrent methods of the financial markets. The daily values of currency pairs for the period from September 2015 to November 2019 were chosen as information basis for monitoring and modelling. The use of $R / S$ modelling method make it possible to conclude the persistence of time series of the selected cryptocurrencies indicating that the market trends are clearly defined, the currency pair of XRP/USD has the highest level of trend resistance. To compare the obtained results, the comprehensive approach is offered using recurrent diagrams that help to determine the cryptocurrency stability. The results of modelling by the recurrent method show that the most stable cryptocurrencies are the ones with the highest capitalization, namely Bitcoin and Ripple.
\end{abstract}

\section{Introduction}

In the context of digitalization and virtualization of the financial sphere, the problem of forecasting the cryptocurrency market dynamically developing in recent years, is of particular importance. The improvement of tools for modelling and forecasting of the cryptocurrency market and risk management are necessary in the context of public distrust of cryptocurrency as an innovative financial asset, and the presence of a number of risks inherent in the cryptocurrency transactions (asset security, hedging complexity, high price volatility, lack of guarantees, legal barriers, the limited use as a means of payment).

National and international researches pay great attention to this problem. Authors [1] point out that the formation and development of the cryptocurrency market are associated with Bitcoin release and blockchain technology that have some spheres of application; and cryptocurrencies are innovative financial asset that attracts investors.

Different approaches and methods are used for modelling and forecasting the cryptocurrency market and forming the investment portfolio. As this market becomes more and more difficult, the potential investors need tools allowing them to form a highly profitable investment portfolio that may include such an asset as cryptocurrency.
To calculate the cryptocurrency market efficiency, paper [2] offers to use Factor Augmented VAR (TVPFAVAR)-model considering the impact of a large number of variable factors on a dependent variable and allowing to study the dynamics of more than 45 cryptocurrencies. This approach helps to conclude that the periods of high/low uncertainty in the market correspond to strong/weak link. The authors explain the trend by the increased degree of the market uncertainty associated with the process of cryptocurrency price fluctuations. In this situation, they propose to form a dual investment portfolio, the structure of which can be varied by the dynamic hedge ratio and the dynamic weights of the portfolio. Using the ARFIMA-FIGARCH model with two distributions and the modified logarithmic periodogram method, authors [3] studied the stability of eight biggest cryptocurrency markets and made a conclusion that they were unstable, volatile, had the limited trading horizons and time gaps, complicating the process of attracting investors.

Paper [4] proposes to assess the dynamics of the cryptocurrency profitability and stability by the multivariate stochastic model, which allows to calculate the average currency volatility. Its practical application shows the significant impact of such factors as the volatility of the cryptocurrency market on the one hand, and the rapid growth in demand for it on the other.

In their paper [5], researchers use a Lotka-Volterra model with variable intervals to model the number of 
transactions for Bitcoin, Litecoin and Ripple using twoand three-dimensional models that allow them to get highprecision forecast for Bitcoin and satisfactory level for Litecoin and Ripple.

The investigation of cryptocurrency time series using econometric models of stochastic volatility is shown in papers $[6,7]$. Alternative one-dimensional dynamic linear and multivariate vector autoregressive models are compared in paper [6]. This approach improves the accuracy of the forecast.

The paper of scientists [8] shows that the use of standard GARCH models can make incorrect VAR and ES forecasts, and thus, lead to inefficient risk management and optimization of the investment portfolio. Therefore, the authors propose to use the method of model building (model complex) of VAR and ES recheck based on a confidence model (MCS) to minimize risks and financial losses.

The traditional method of the cryptocurrency market analysis is technical analysis, but in the conditions of high market volatility, it is reasonable to combine the methods of technical analysis with the methods of economic and mathematical modelling. Thus, authors [9] propose a nonparametric model based on technical analysis as an alternative method of assessment and forecasting of the cryptocurrency market. This approach calculates the forecast values of Bitcoin's profitability through a neural network and indicates the speculative nature of the market.

Traditional methods and modelling do not allow to make accurate forecasts and calculations for the development of the cryptocurrency market being a nonlinear complex economic system and to detect the occurrence of crisis phenomena.

Therefore, it is necessary to use the methods being traditionally inherent in other fields of science. Econophysics has rather powerful methodological apparatus for modelling complex socio-economic systems in modern economic science.

Thus, paper [10] points out that it is necessary to apply comprehensive approach for the forecast considering the nonlinear dynamics and the inherent chaos and fractality of the digital currencies. The authors propose the hybrid model for the forecast based on the neural network of long-term memory (LSTM) and empirical wavelet decomposition (EWT), along with the cuckoo search algorithm (CS) for digital currency time series to obtain more accurate forecast values.

Authors [11] use monofractal analysis to investigate the price volatility in the cryptocurrency market and multifractal fluctuation analysis to test the model for stability.

A strong impulse effect in the Bitcoin and Ethereum markets and a reversal effect for Ripple and EOS at high fluctuations were found as a result of the model implementation. The application of this model will help to form effective alternative strategies for the allocation of assets in the investment portfolio.

Ukrainian scientists [12] propose the procedure for determining the normalized economic coordinates, economic mass and heterogeneous economic time, based on the basic concepts of general theory of relativity and relativistic quantum mechanics. They are based on the analysis of time series describing socio-economic phenomena and economic interpretation of uncertainty by Heisenberg. The authors confirm that the economic mass of the time series can be an indicator of crisis phenomena.

Therefore, a combination use of different methods of economic and mathematical modelling of the cryptocurrency market allows to increase the accuracy of forecasting, to identify the indicators of crisis phenomena, to analyze the degree of volatility and risk of both individual cryptocurrencies and their pairs.

\section{Research methodology}

\subsection{R/S-analysis}

$R / S$-analysis was first proposed by B. Mandelbrot [13] and is based on H. Hurst hydrological studies. According to Hurst, the essence of fractal analysis for time series is to process the structure of a series that reflects a certain process and demonstrates a quantitative degree of chance. In paper [14], this approach was proposed to quantify the nature of self-affine structures.

The method of $R / S$-analysis allows calculating the parameter of self-similarity $H$, which measures the intensity of long-term dependencies in a time series. This metric is based on the analysis of the parameter range and the standard deviation.

Here is an algorithm for $R / S$-analysis. Suppose that the time series $y=\left\{y_{i}\right\}, i=1,2, \ldots, N$ is given, which must be led to "logarithmic returns". The resulting sequence is divided into initial segments $y=y_{1}, y_{2}, \ldots, y_{N}$.

(i) The average value and standard deviation of $S_{n}$ are calculated for each segment.

(ii) The cumulative deviation is calculated:

$$
x_{t, N}=\sum_{i=1}^{t}\left(y_{i}-M_{N}\right),
$$

where $y_{i}$ - increase in a year $i, M_{N}$ - average, $y_{i}$ for $N$ periods.

(iii) Range is determined:

$$
R(N)=\max \left(x_{t, N}\right)-\min \left(x_{t, N}\right),
$$

where $R(N)$ - deviation range $x_{t, N}, \max \left(x_{t, N}\right)$ - maximum value for $x_{t, N}, \min \left(x_{t, N}\right)-$ minimum value for $x_{t, N}$.

(iv) From the ratio

$$
R / S=(a N)^{H},
$$

where $R / S$ - normalized range, $N$ - observation number, $a$ - constant, the Hurst exponent $H$ is derived.

By the value of the Hurst coefficient, we can identify time series and draw conclusions about the minimum forecast of these series behaviour:

(i) $H=0.5$ - the sequence is white noise; the time series is random. The future values of this series are independent of the previous ones. System status is random walk;

(ii) $0<H<0.5$ - anti-persistent or ergodic time series (pink noise), i.e. a series characterized by the so-called "return to average": if there is a decline in the indicator in 
the studied system over a period of time, an increase should be expected in the next period. The closer $H$ is to 0 , the more variable the series is. These changes happen quickly, often, but they are small. Note that such processes are few in reality. System status - flute;

(iii) $0.5<H<1-$ persistent or trend resistant time series (black noise, Brownian motion). The time series is characterized by the effect of long-term memory. If the series is growing, it is likely to continue to grow. If it falls today, a decline should be expected tomorrow. The closer the value of $H$ is to 1 , the more trend resistance is. The closer the value of $N$ is to 0.5 , the noisier the number will be. System status is a trend.

\subsection{Recurrent diagrams}

Recurrent diagrams as a graphical tool are proposed in [15]. They are based on the theory of phase reconstruction of space. Many processes in nature are known to have periodical character, i.e. recurrent behaviour. Moreover, the recurrence (repeatability) of states in the meaning of passing a further trajectory close enough to the previous one is a fundamental property of dissipative dynamic systems [16]. A. Poincare discovered this property and formulated it in the form of a "recurrence theorem": if the system reduces its dynamics to a limited subset of the phase space, it (the system) is almost certain, i.e., with a probability, almost equals to 1 , as close to any initially set mode as possible [17].

The content of this theorem is that a complex dynamical system can deviate from its state by the exponential law due to the smallest perturbation, but after a while the system will seek to return to the initial state and undergo similar stages of evolution.

J. P. Eckmann and co-authors [15] propose a method of displaying $m$ - the measured phase trajectory of system states $\vec{x}(t)$ of $N$ length, on a two-dimensional square binary matrix of $N \times N$ size, in which 1 (black point) corresponds to the repetition of the state at some time $i$ at some other time $j$; and both coordinate axes are time axes. This display was called a recurrent diagram because it captures information about the recurrent behaviour of the system (Table 1).

Formally, the recurrent diagram can be expressed by the matrix:

$$
R_{i, j}^{m, \varepsilon_{i}}=\Theta\left(\varepsilon_{i}-\left\|\vec{x}_{i}-\vec{x}_{j}\right\|\right), \vec{x} \in \mathfrak{R}^{m}, \cdot i, j=1, \ldots, N
$$

where $N$ - the number of measured points, $\overrightarrow{x_{l}}, \varepsilon_{i}$ - the size of neighbourhood of point $\vec{x}$ at a moment $i$, $\|\cdot\|$ - normalization operator and $\Theta(\cdot)-$ the Heaviside function.

Since $R_{i, j}=1(i=1, \ldots, N)$ by definition, the recurrent diagram always contains a diagonal line consisting of black points - an identity line at an angle of $\pi / 4$ to the coordinate axes. A separate recurrent point does not provide useful information about states in time $i$ and $j$. Only the whole set of recurrent points allows to restore the properties of the system.

Visual assessment of recurrent diagrams can give an idea of the nature of the processes occurring in the system under study and conclude that the state of change is rapidly due to critical phenomena.

Table 1. Typical samples of recurrent diagrams and their interpretation [16].

\begin{tabular}{|l|l|}
\hline \multicolumn{1}{|c|}{ Sample } & \multicolumn{1}{c|}{ Interpretation } \\
\hline Homogeneous & Stationary process \\
\hline $\begin{array}{l}\text { Extinction in the } \\
\text { upper left and } \\
\text { lower rights } \\
\text { corners }\end{array}$ & $\begin{array}{l}\text { Non-stationary data; the process contains } \\
\text { trend or drift }\end{array}$ \\
\hline $\begin{array}{l}\text { Destruction (white } \\
\text { stripes) }\end{array}$ & $\begin{array}{l}\text { Non-stationary data; some conditions are } \\
\text { exceptional or far from normal; } \\
\text { transitions may have occurred }\end{array}$ \\
\hline $\begin{array}{l}\text { Periodic / } \\
\text { quasiperiodic } \\
\text { samples }\end{array}$ & $\begin{array}{l}\text { Cyclicity in the process; the time distance } \\
\text { between periodic samples (e.g. lines) } \\
\text { corresponds to the period; the difference } \\
\text { in distances between long diagonal lines } \\
\text { shows the quasi-periodicity of the process }\end{array}$ \\
\hline $\begin{array}{l}\text { Diagonal lines } \\
\text { (orthogonal to the } \\
\text { main diagonal) }\end{array}$ & $\begin{array}{l}\text { The evolution of a state is similar to time } \\
\text { difference }\end{array}$ \\
\hline $\begin{array}{l}\text { Vertical / } \\
\text { horizontal lines } \\
\text { (clusters) }\end{array}$ & $\begin{array}{l}\text { Some states do not change or change over } \\
\text { time; sign of laminar states }\end{array}$ \\
\hline $\begin{array}{l}\text { Long inclined } \\
\text { structural lines }\end{array}$ & $\begin{array}{l}\text { The evolution of states is similar in } \\
\text { different periods, but at different speeds; } \\
\text { the dynamics of systems could change }\end{array}$ \\
\hline
\end{tabular}

\section{Research findings}

Six cryptocurrencies were selected for the study; they differ in cryptographic (software) code. They are Bitcoin (BTC, forks), Namecoin (NMC, forks), Ethereum (ETN, crypto 2.0 - the second-generation cryptocurrencies), BitShares (BTS, crypto 2.0 - the second-generation cryptocurrencies), Ripple (XRP, code from scratch), Nextcoin (NXT, code from scratch). Bitcoin, Ethereum, Ripple cryptocurrencies are at the top of their capitalization ratings. Other cryptocurrencies used in the paper are less capitalized, but they are within 450 positions in the ranking. The study covers the time span from September 2015 to November 2019. The calculations were made in the MatLab environment based data [18].

Here are the estimated values of the Hurst indicator for cryptocurrency pairs (Table 2).

Table 2. Results of the Hurst coefficient calculations.

\begin{tabular}{|c|c|}
\hline Currency pair & Hurst coefficient value $(\boldsymbol{H})$ \\
\hline XRP/USD & 0.744 \\
\hline ETH/USD & 0.708 \\
\hline NXT/USD & 0.692 \\
\hline BTS/USD & 0.678 \\
\hline BTC/USD & 0.637 \\
\hline NMC/USD & 0.571 \\
\hline
\end{tabular}

According to the obtained values of the Hurst coefficient (Table 2), we can conclude that the time series of these cryptocurrencies are persistent, i.e. the markets show clear trends, since the values of the Hurst coefficient are in the range of 0.5 to 1 . The XRP/USD currency pair 
has the highest $H=0.74$ meaning that it has the highest trend resistance among other currency pairs. In our view, the legal recognition of this cryptocurrency at the state level is an influential factor. It is one of the most popular cryptocurrencies today. It was created to accelerate transactions and currency conversion; it is also one of the cryptocurrencies used to support the technology of the future "Internet of Things".

Table 2 shows that the minimum value $H=0.571$ has the NMC/USD currency pair indicating its least trend resistance. This cryptocurrency has one of the lowest levels of capitalization among the cryptocurrencies under consideration.

Fig. 1 shows the results of calculations in the form of recurrent maps. The analysis of recurrent diagram reveals common features in distributions of both topology and structure of diagrams. The dark areas of the diagram indicate that the series is relatively stable. It should be noted that the irregular appearance of black and white zones indicates the irregularity of the processes occurring in the system (Fig. 1 e).
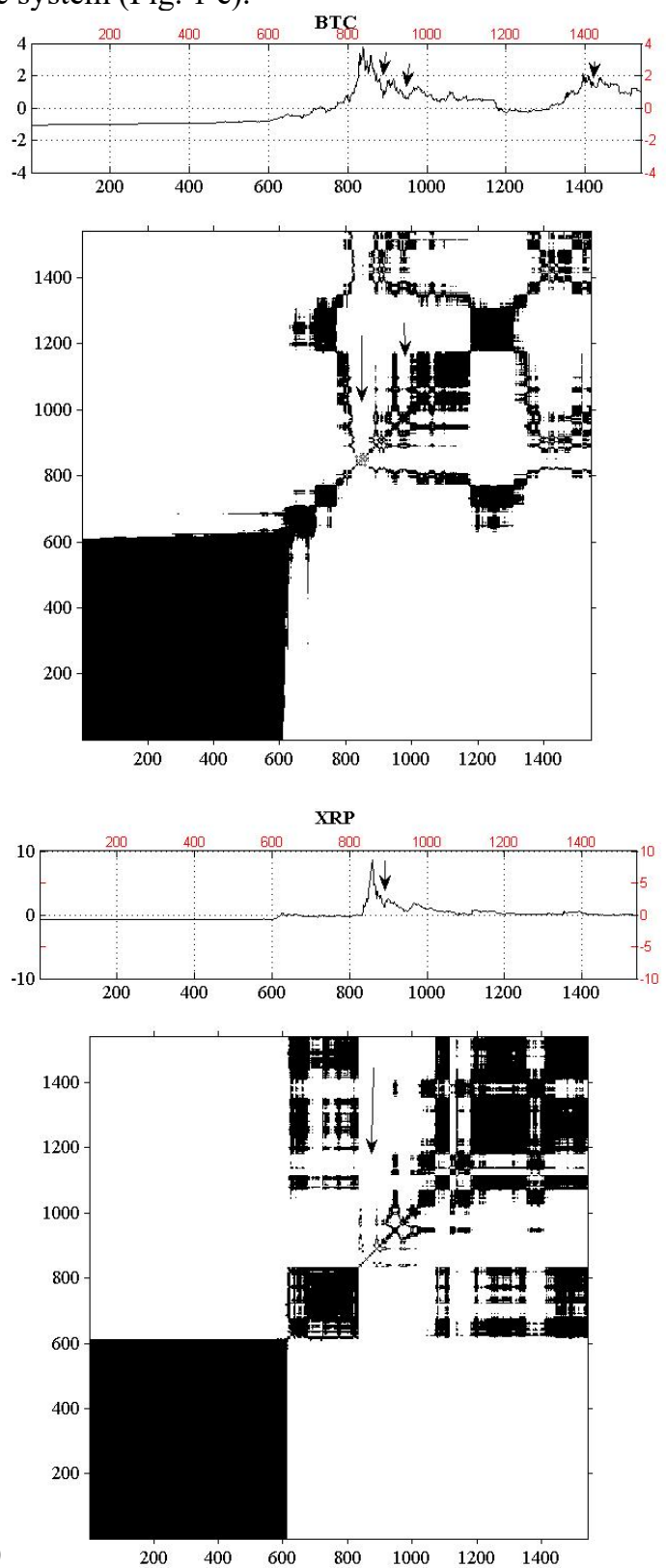

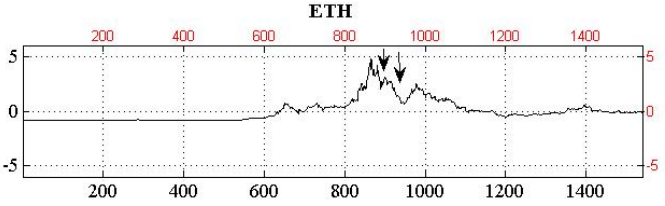

c)
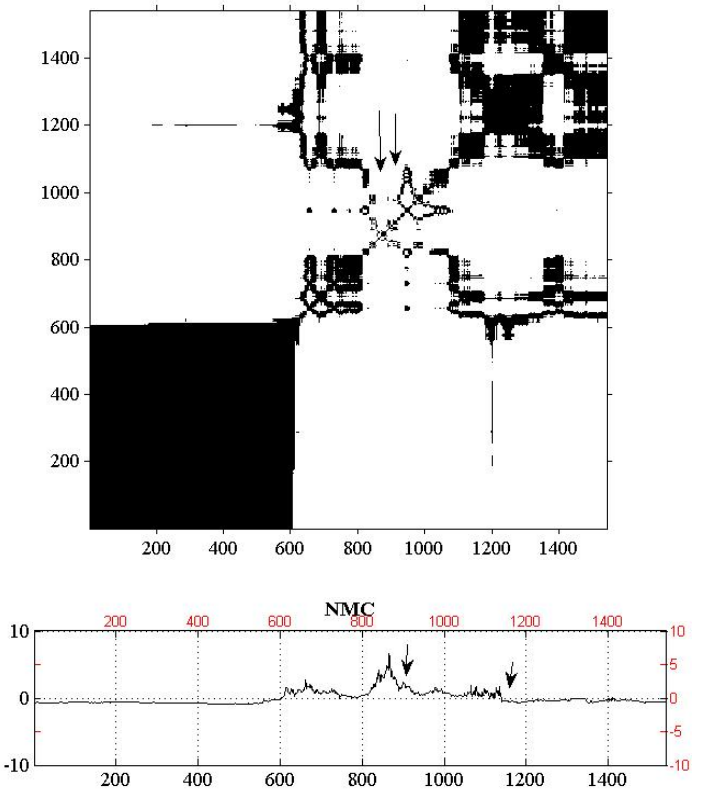

d)
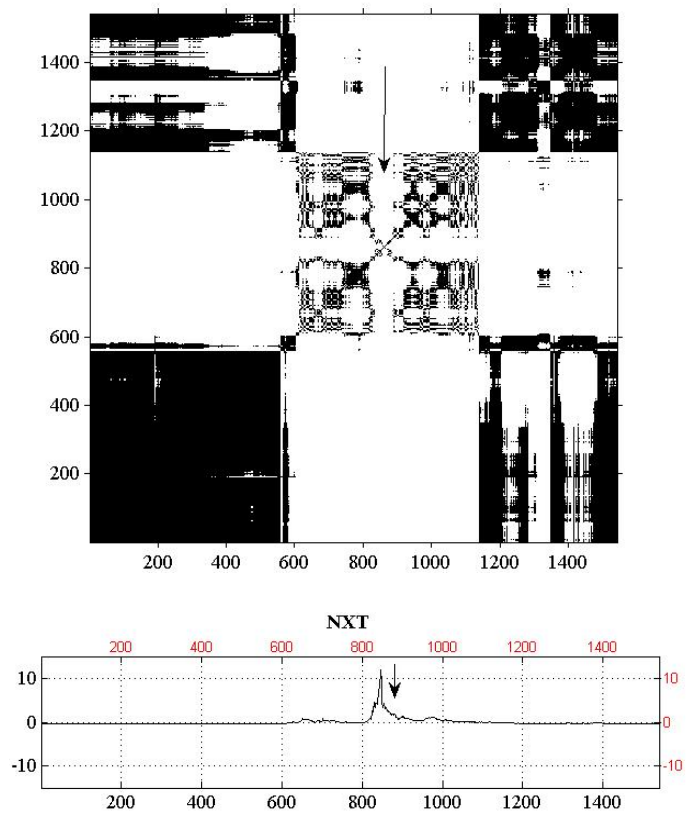

e)

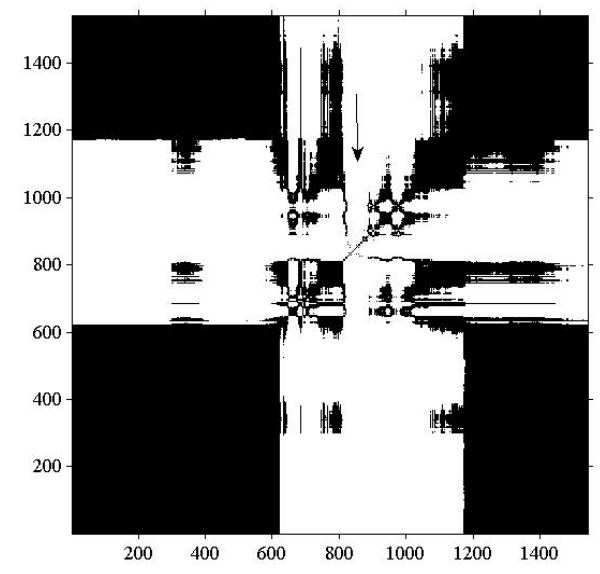



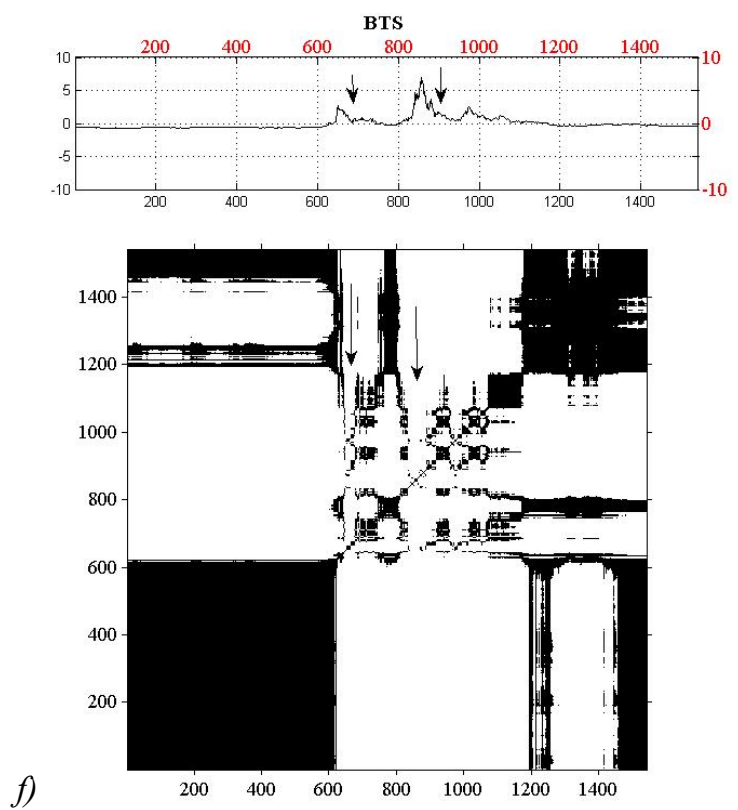

Fig. 1. Recurrent time series diagrams of Bitcoin (a), Ripple (b), Ethereum (c), Namecoin (d), Nextcoin (e), BitShares (f).

At the same time, we can state that the points are mostly located along the diagonal line for the series having higher values of the Hurst coefficient. The recurrent points fill a larger region of phase space for series with a small Hurst value.

Fig. 1 shows that in the neighbourhood of point 900 (2018 crisis), recurrent diagrams for cryptocurrency time series are starting to change. For the studied currency pairs, we observe the appearance of white areas and stripes during a crisis in the neighbourhood of point 900. It can also be noted that the recurrent diagrams for more capitalized currencies is similar in nature, especially for the recurrent diagrams of Bitcoin cryptocurrencies (Fig. 1 a), Ripple (Fig. 1 b), and Ethereum (Fig. 1 c).

The recurrent diagrams are somewhat different for the time series of Nextcoin cryptocurrencies (Fig. 1 e), and BitShares (Fig. 1 f). The recurrent diagrams of these cryptocurrencies are characterized by frequent changes of white and dark areas. Concerning the time series of Namecoin cryptocurrency (Fig. 1d), its recurrent diagram is similar to the recurrent diagrams of the most capitalized cryptocurrencies. However, there are white spots and lines in the beginning of 2019 (1200 point). It shows a certain crisis situation of these cryptocurrencies during that period.

\section{Conclusions}

We can conclude based on the results of the cryptocurrency market research that cryptocurrencies have long-term potential and prospects in today's globalized economy being subject to crisis.

The $R / S$-analysis shows that trend-resistant cryptocurrencies are the most capitalized in times of crisis. Ripple, the cryptocurrency being the official payment instrument in Japan since 2017, is marked by its stability.
A comprehensive approach to monitoring and modelling the cryptocurrency market using recurrent diagrams provides information on the temporal correlation of phase space points and determines the status and trends of the cryptocurrency markets with sufficient accuracy regardless of their classification. The analysis of the cryptocurrency market in the paper leads to the conclusion that the state of currencies on it is quite stable at the current date.

The proposed methodology for monitoring and modelling the cryptocurrency market is of practical importance as it will allow potential investors to form a profitable portfolio with a high level of reliability and stability over time.

\section{References}

1. A. Berentsen, F. Schar, A Short Introduction to the World of Cryptocurrencies. Review 100(1), 1-16 (2018). doi: 10.20955/r.2018.1-16

2. N. Antonakakis, I. Chatziantoniou, D. Gabauerac, Cryptocurrency market contagion: Market uncertainty, market complexity, and dynamic portfolios. Journal of International Financial Markets, Institutions and Money 61, 37-51 (2019). doi:10.1016/j.intfin.2019.02.003

3. M. Omane-Adjepong, P. Alagidede, N. Kwame Akosah, Wavelet time-scale persistence analysis of cryptocurrency market returns and volatility. Physica A: Statistical Mechanics and its Applications 514 (2019). doi:10.1016/j.physa.2018.09.013

4. P. Chaim, M.P. Laurini, Nonlinear dependence in cryptocurrency markets. The North American Journal of Economics and Finance 48, 32-47 (2019). doi:10.1016/j.najef.2019.01.015

5. P. Gatabazi, J. C. Mba, E. Pindza, Modeling cryptocurrencies transaction counts using variableorder Fractional Grey Lotka-Volterra dynamical system. Chaos, Solitons \& Fractals 127, 283-290 (2019). doi:10.1016/j.chaos.2019.07.003

6. L. Catania, S. Grassi, F. Ravazzolo, Forecasting cryptocurrencies under model and parameter instability. International Journal of Forecasting 35(2), 485-501 (2019). doi:10.1016/j.ijforecast.2018.09.005

7. A. Phillip, J. Chan, S. Peiris, On generalized bivariate Student-t Gegenbauer long memory stochastic volatility models with leverage: Bayesian forecasting of cryptocurrencies with a focus on Bitcoin. Econometrics and Statistics (2018). doi:10.1016/j.ecosta.2018.10.003

8. G.M. Caporale, T. Zekokh, Modelling volatility of cryptocurrencies using Markov-Switching GARCH models. Research in International Business and Finance 48, 143-155 (2019). doi:10.1016/j.ribaf.2018.12.009

9. R. Adcock, N. Gradojevic, Non-fundamental, nonparametric Bitcoin forecasting. Physica A: Statistical Mechanics and its Applications 531 (2019). doi:10.1016/j.physa.2019.121727 
10. A. Altana, S. Karasua, S. Bekiros, Digital currency forecasting with chaotic meta-heuristic bio-inspired signal processing techniques. Chaos, Solitons \& Fractals 126, 325-336 (2019). doi:10.1016/j.chaos.2019.07.011

11. Q. Chenga, X. Liub, X. Zhub, Cryptocurrency momentum effect: DFA and MF-DFA analysis. Physica A: Statistical Mechanics and its Applications. 526 (2019). doi:10.1016/j.physa.2019.04.083

12. A. Danil`chuk, V. Solov`ev, Ispol`zovanie princzipa neopredelennosti Gejzenberga dlya modelirovaniya krizisny`kh yavlenij na ry`nke kriptovalyut. E’konomicheskie i finansovy`e mekhanizmy` innovaczionnogo razvitiya czifrovoj e konomiki (Use of the Heisenberg uncertainty principle to model crisis phenomena in the cryptocurrency market). (Institut biznesa BGU, Minsk, 2019), pp. 172-177

13. B.B. Mandelbrot, Robustness of the rescaled range $\mathrm{R} / \mathrm{S}$ un the measurement dependence. Water Resources Research 5(5), 967-988 (1969)

14. H.E. Hurst, Long-Term Storage Capacity of Reservoirs. Transactions of the American Society of Civil Engineers 116(1), 770-799 (1951)

15. J.P. Eckmann, S. Kamphorst, D. Ruelle, Recurrence plots of dynamical systems. Europhys Lett. 4(9), 973977 (1987)

16. V.M. Soloviov, V.D. Derbentsev, O.A. Serdiuk, O. D. Sharapov, Synerhetychni ta ekonofizychni metody doslidzhennia dynamichnykh ta strukturnykh kharakterystyk ekonomichnykh system (Synergetic and econophysical methods for the study of dynamic and structural characteristics of economic systems). (Brama-Ukraina, Cherkasy, 2010)

17. H. Poincaré, Sur le problème des trois corps et les équations de la dynamique. Acta Mathematica 13, 1270 (1890)

18. Yahoo Finance. (2019), https://finance.yahoo.com. Accessed 30 Oct 2019 